\title{
Linx
}

Revue des linguistes de l'université Paris X Nanterre

47 | 2002

Du sens au sens

\section{Les titres des Fables de la Fontaine : « définitude », généricité, narrativité}

On the titles of La Fontaine's Fables : definiteness, genericity, narrativeness

\section{Jacques Anis}

\section{OpenEdition}

\section{Journals}

Édition électronique

URL : http://journals.openedition.org/linx/559

DOI : $10.4000 /$ linx. 559

ISSN : 2118-9692

\section{Éditeur}

Presses universitaires de Paris Nanterre

\section{Édition imprimée}

Date de publication : 1 décembre 2002

Pagination : 31-42

ISSN : 0246-8743

\section{Référence électronique}

Jacques Anis, "Les titres des Fables de la Fontaine : « définitude », généricité, narrativité », Linx [En ligne], 47 | 2002, mis en ligne le 01 juin 2003, consulté le 30 avril 2019. URL : http:// journals.openedition.org/linx/559; DOI : 10.4000/linx.559 


\title{
Les titres des Fables de la Fontaine : « définitude ", généricité, narrativité
}

\author{
Jacques Anis, Paris X - Nanterre, Modyco, UMR 7114 Paris X/CNRS
}

Ayant entamé récemment une recherche sur les titres de poèmes dont le déclencheur initial fut une étude sur Francis Ponge (Anis, 2002) et qui se concrétise à l'heure actuelle par une étude des titres des fables de La Fontaine, il m'a semblé tout à fait naturel de saisir l'occasion de cet hommage à un ami très cher, sémanticien de haute volée, pour approfondir la problématique de la détermination nominale, en m'appuyant notamment sur ses travaux. C'est à un de ses articles (Galmiche, 1989) que j’emprunte le terme de définitude 1 , et la généricité a été l'objet de plusieurs de ses publications (Galmiche, 1983, 1985 et Galmiche et Kleiber (éds), 1985)².

\section{Introduction}

\subsection{Qu'est-ce qu'un titre?}

Dans un ouvrage consacré aux titres des tableaux, Bosredon rattache l'intitulation à la pratique de l'étiquetage défini comme «une pratique langagière spécifique par laquelle un objet in prasentia est identifié au moyen d'une séquence linguistique écrite qui lui est contiguë» (1997 : 14). Ce cadrage général une fois retenu, on notera qu'il existe cependant une différence majeure entre le titre de tableau et le titre de poème : dans ce dernier cas le titre est de même nature que le texte.

Dans son célèbre traité sur l'article, Guillaume consacre près de deux pages aux

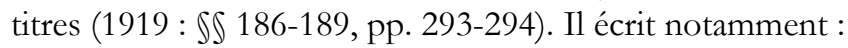

\footnotetext{
${ }^{1}$ Notons aussi que le premier numéro de Linx était essentiellement constitué par son article sur la description définie (1979)!

${ }^{2}$ Je remercie chaleureusement D. Leeman pour ses critiques amicales autant qu'acérées et en tout cas fort constructives. J'ai tenté également de tirer profit des remarques précieuses des relecteurs anonymes.
} 
Jacques Anis

Le fait général sur lequel se fonde leur syntaxe est que le titre étant placé sur l'objet même, une concurrence est possible entre la vision réelle, action des sens, et la vision linguistique, action de l'esprit.

Le plus haut degré de cette concurrence est représenté par le cas des étiquettes. Un écriteau «œufs» étant posé sur des œufs, mon esprit réalisera l'image, par contemplation de chose et non par contemplation d'idée (op. cit: 293) ${ }^{3}$.

L'auteur oppose ensuite les «titres formels», qui « désignent l'objet par son enveloppe extérieure » et sont "introduits par zéro» et les «titres matériels », qui « désignent l'objet par son contenu intérieur » et « se font précéder de l'article » (ibid.).

Il prend d'ailleurs l'exemple de La Fontaine, dont les recueils présentent en général une double titulature : «Fable XI. Le lion et le rat» (II, 9), le premier élément, formel, répondant à la question " qu'est-ce que ceci ? ", le second indiquant le sujet de la fable.

Le lecteur se dira peut-être à ce stade que la question a été magistralement traitée et qu'il est inutile d'aller plus loin. Mais notre illustre prédécesseur ne traite pas le choix prédominant de l'article défini. Plus fondamentalement, la question que nous voulons approfondir nécessite à notre sens une prise en compte de facteurs logicosémantiques - statut métalinguistique du titre, question de la référence, problème de la généricité - et textuels - rôle d'incipit et de synopsis du récit.

\subsection{Constitution $d u$ corpus}

De l'édition de référence $[1990]^{4}$, nous avons écarté les divers textes, en prose ou en vers, à caractère dédicatoire ou introductif, pour ne garder que les textes comportant une double titulature, le premier titre étant toujours Fable $<\mathrm{N}>$, par exemple «Fable I. La Cigale et la Fourmi $»^{5}$. Nous ne prenons pas non plus en compte les fables non intégrées au recueil ou posthumes. Le corpus se compose donc de 241 doubles titres.

\section{La pluri-fonctionnalité et la polyphonie des titres}

\subsection{La fonction d'identification}

En tant qu'étiquette, le titre est un quasi-nom propre, qui dénomme le texte in situ et permet ensuite l'acte de langage de référence ${ }^{6}$. Le titre doit donc idéalement être singulier, pour permettre l'identification du référent, il s'agit d'une description définie ${ }^{7}$.

\footnotetext{
${ }^{3} \mathrm{Si}$ on reprend le cas du tableau, la prédominance notée par Bosredon de l'article zéro pour les titres des tableaux figuratifs s'expliquera par la prégnance de la contemplation de l'analogon de la chose.

${ }^{4}$ Voir seconde partie des références bibliographiques. Cette édition comportant quelques défauts ponctuo-typographiques, nous la corrigeons parfois d'après celle de 1966.

${ }^{5}$ Nous référencerons maintenant les fables selon le modèle « N, N’ (livre, fable).

${ }^{6}$ Nous nous inspirons assez librement de Searle (1972: 115-142).

${ }^{7}$ Voir pour un rappel de cette problématique Galmiche (1989 : 8-12), Searle (op. cit. : 125-126).
} 
On peut ainsi évoquer La Chartreuse de Parme ou Le Père Goriot. On notera toutefois que lorsque le titre est formel au sens de Guillaume, la construction de la référence exige de le compléter par le nom de l'auteur: les Fables d'Ésope, les Fables de Phèdre, les Fables de La Fontaine. Certains titres matériels n'échappent pas à cette difficulté : Médée d'Euripide, Médée de Sénèque ; Antigone de Sophocle, Antigone d'Anouilh. On notera que lorsque, comme dans le cas de La Fontaine, l'œuvre est très connue, l'expression prend pratiquement le statut d'un titre.

Pour ce qui nous intéresse, à savoir les titres matériels des fables, certains reprennent littéralement, si l'on néglige le phénomène de la traduction, ceux de Phèdre : Le Loup et l'Agneau (I, 10), Le Renard et la Cigogne (I, 18).

Dans la fonction identificatrice, le titre formel serait insuffisant, car la numérotation se fait dans le cadre de chaque livre. Il faut donc dire « la fable I du livre $\mathrm{X}$ des Fables de La Fontaine ", expression réservée aux textes savants. On peut considérer que le titre formel a comme identificateur une portée purement locale, ne fonctionnant que comme une balise dans l'espace graphique du livre alors que le titre matériel est d'usage général. Il s'inscrit dans la mémoire individuelle et collective des francophones.

Dans le discours, le titre fonctionne en mention et peut entrer dans la catégorie de l'îlot textuel ${ }^{8}$.

\subsection{Fonction thématique}

L'objet privilégié de cette étude est évidemment le titre matériel de la fable. Celui-ci, in situ, peut être considéré comme intra-textuel aussi bien que méta-textuel ou paratextuel. Pourquoi ne pas en faire un incipit? Il serait peut-être possible de considérer le titre comme le thème et le corps comme le propos ${ }^{9}$. De fait le texte répondrait à la question "Qu'est-il arrivé à la cigale et à la fourmi ? » On peut aussi reprendre la notion de matrice textuelle proposée par Riffaterre $(1983,1989)$ et faire du corps de la fable l'expansion du titre.

Symétriquement, le titre peut aussi être envisagé comme un résumé anticipé $\mathrm{du}$ texte, avec lequel il entretient des relations paraphrastiques de condensation/expansion.

Ce qui est spécialement illustré par des titres comportant la mention d'un personnage et une évocation de l'action de la fable, sous la forme d'un SN et d'un modificateur, par exemple I, 3 et V, 21 :

\section{Fable III. La Grenouille qui se veut faire aussi grosse que le Bœuf}

Une Grenouille vit un bœuf

Qui lui sembla de belle taille.

\footnotetext{
${ }^{8}$ Sur cette notion, on peut lire notamment Authier-Revuz (1996) et retenir sa définition : « fragment textuel du message $(\mathrm{m})$ de 1 [le locuteur «autre»] ayant "résisté dans sa littéralité à l'opérationtraduction à l'œuvre dans le message M [émanant du locuteur] » (p. 94).

${ }^{9}$ Nous reprenons ici une suggestion de Carole Tisset (communication personnelle).
} 
Jacques Anis

Elle qui n'était pas grosse en tout comme un œuf,

Envieuse s'étend, et s'enfle, et se travaille

Pour égaler l'animal en grosseur...

Fable VI. L'Oiseau blessé d'une flèche

Mortellement atteint d'une flèche empennée,

Un Oiseau déplorait sa triste destinée...

\subsection{Polyphonie}

Le titre, de par la coexistence des fonctions, fait l'objet d'une lecture à plusieurs niveaux ${ }^{10}$. En ce sens, la détermination nominale dépend à la fois du niveau métatextuel ou para-textuel et du niveau textuel. Pour symboliser cela, on pourrait proposer de voir dans Le Chat et le Renard (IX, 14) la réalisation superficielle d'une structure profonde qui serait LE [Le chat et le renard]. Ce LE sous-jacent exprimerait par sa définitude l'existence et la singularité de l'objet textuel fable.

\subsection{Intertextualité}

Dans la ligne de ce que nous avons dit plus haut, on peut parler d'intertextualité entre le titre et le texte, car aux paraphrases s'ajoutent des reprises littérales.

Le début d'une des fables les plus célèbres $(V, 10)$ illustre ce point :

Fable X. Le Lièvre et la Tortue

Rien ne sert de courir ; il faut partir à point.

Le Lièvre et la Tortue en sont un témoignage.

Gageons, dit celle-ci...

S'ajoute une intertextualité externe affichée, puisque La Fontaine, selon l'esthétique classique, se bornerait à «mettre en vers » les fables d'Ésope, de Pilpay, et de quelques autres. Le terme de fable était d'ailleurs très extensif et désignait des formes diverses de récit légendaire, en prose ou en vers. Ainsi on trouvera Philémon et Baucis (XII, 27) - avec le sous-titre «Sujet tiré des métamorphoses d'Ovide » - et La Matrone d'Éphèse (XII, 28) (épisode du Satiricon) - avec une mention explicite de l'auteur antique dans le corps du texte ${ }^{11}$. Ces titres-là, comme ceux des fables reprises de Phèdre - Le loup et l'agneau (I, 10), Le Renard et la Cigogne (I, 18) - font des fables en question des bypertextes au sens de Genette, à savoir des réécritures plus ou moins directes de textes préexistants dits bypotextes.

\footnotetext{
${ }^{10}$ Nous utilisons ici cette notion de manière extensive, sans doute trop pour certains spécialistes...
}

11 vv. 6-7 : «Quelle grâce aura ta Matrone/Au prix de celle de Pétrone? » 


\section{La détermination nominale : la prédominance de l'article défini}

Il n'existe dans le corpus que deux exemples de titres ${ }^{12}$ ne constituant pas un syntagme nominal. On peut voir en ceux-ci des modificateurs du premier titre. Contre ceux qui ont le goût difficile (II, I) est un titre purement métatextuel, et la fable elle-même est atypique, car elle est construite en abîme: le poète se met lui-même en scène, traitant pour plaire à des critiques l'apologue du Cheval de Troie, et arrêté par eux pour une rime pauvre. Du Thésauriseur et du Singe (XII, 3) reprend le modèle titulaire hérité du latin.

Les 239 restants sont constitués d'un ou plusieurs SN. Les 77 (32\% environ) qui n'en contiennent qu'un peuvent être qualifiés d'uni-nucléaires, 162 sont polynucléaires, qui se répartissent ainsi : 137 bi-nucléaires (57\%), 23 tri-nucléaires $(10 \%), 2$ quadri-nucléaires $(1 \%)$.

Nous restreignons le corpus de départ aux titres uninucléaires, et nous sélectionnons les 74 qui ont comme noyau un nom commun ${ }^{13}$.

\subsection{L'article zéro}

Parmi les quatre titres comportant l'article zéro, on trouve un titre formel : la fable X, 14 présente en effet la succession atypique de deux titres formels, à savoir Fable XIV. Discours. Dans notre édition, la dédicace «A Monsieur le duc de la Rochefoucauld» est malencontreusement intégrée typographiquement au corps du texte, mais dans celle de 1966 elle fait bloc avec le titre, avec la marque typographique des petites capitales.

Cette bizarrerie s'inscrit dans la difficile intégration parmi les fables de pièces poétiques dédiées. Ainsi dans notre édition, le Discours à Madame de la Sablière est suivi au livre IX de la Fable XX Les deux Rats, le Renard, et l'cuf, alors que dans l'édition de 1966, celle-ci y est intégrée avec le même intitulé en intertitre ${ }^{14}$.

Les titres Testament expliqué par Ésope (II, 20) et Parole de Socrate (IV, 17) sont intégrés par Guillaume dans la catégorie des «titres duplicatifs » (op. cit., \189, premier sous-paragraphe : 294-295), ainsi définis : «Le titre qui serait formel appliqué à l'objet reste formel lorsqu'on l'applique au double de l'objet: reproduction, copie, etc. » (op. cit. : 295)

L'argumentation ci-dessous nous paraît plus spécieuse, en ce qui concerne le dernier exemple :

\footnotetext{
12 Nous réduisons maintenant le champ au second titre.

13 Pour satisfaire la curiosité du lecteur qui se demande quels sont les titres écartés, précisons que l'un a comme noyau un pronom: Rien de trop (IX, 11) est de nature intertextuelle car c'est la traduction, d'ailleurs reprise en îlot textuel dans la morale, du célèbre précepte antique «mêden agan »; alors que les deux autres comportent des noms propres : Belphégor (XII, 27), de nature explicitement hypertextuelle puisque sous-titré « Nouvelle tirée de Machiavel », et Simonide préservé par les Dieux (I, 14).

14 L'édition de 1679 présentait également comme Discours au duc du Maine la fable intitulée maintenant Les Dieux voulant instruire un fils de Jupiter (maintenant XI, 2). À l'inverse, l'édition de 1872 normalise avec le titre Les lapins la fable X, 14.
} 
Jacques Anis

Il faut rattacher à ce cas des titres sans article comme : Bataille de Bouvines, Lever de soleil, qui, parfaitement justifiés lorsqu'ils sont placés au bas d'un tableau proprement dit, restent admissibles lorsque le tableau n'est que littéraire. On remarquera que les titres de cette dernière catégorie supposent un premier nom qui désigne l'ensemble de l'événement décrit. Ex. : Conseil tenu par les rats (La Font., II, 2). (ibidem)

dans la mesure où nous pensons plutôt qu'il faut s'appuyer sur la relation transformationnelle avec la phrase «Les rats tiennent conseil », où l'article zéro correspond à la détermination canonique du nom.

D'ailleurs, on peut opposer à l'argumentation de Guillaume le titre Le combat des Rats et des Belettes (IV, 6). Il est vrai que dans celui-ci l'article défini est favorisé par le complément de nom. Mais Combat des Rats et des Belettes serait à la rigueur possible.

Le titre de la fable Tribut envoyé par les Animaux à Alexandre (IV, 12) renforce notre hypothèse, dans la mesure où il est écrit aux vers 19-20 : «Après divers avis, on résout, on conclut/D’envoyer hommage et tribut...».

\subsection{L'article indéfini}

Nous n'avons qu'un exemple de titre commençant par un article indéfini. Il s'agit d'une sorte d'îlot textuel, mais qui ne peut se révéler qu'à la lecture de la fable : Un Animal dans la Lune (VII, 19). En effet, la fable ne met pas en scène un animal qui évoluerait sur notre satellite, mais des savants qui, à cause d'une souris prise entre les verres d'un télescope, croient voir « un animal dans la Lune $»^{15}$.

\subsection{L'article défini}

Rappelons que c'est le cas standard : 68 sur nos 76 titres $^{16}$ (près de $90 \%$ ).

Nous avons suggéré plus haut d'attribuer à l'article défini un statut double : il serait pré-sélectionné par l'unicité de chaque fable. Ce n'est pas une règle absolue, mais une tendance très forte, confirmée si l'on regarde les titres binucléaires - près de $92 \%$ - et les tri- et quadrilinéaires - 100\% !

Le calcul interprétatif ${ }^{17}$ de la définitude dépend de l'interaction de trois sources : le contenu linguistique intrinsèque du titre, le rapport titre-texte - le $\mathrm{N}$ dont on va parler - et le contenu du texte. Ces trois sources sont asynchrones : la première immédiate, la seconde anticipatrice, la troisième rétroactive.

${ }^{15}$ En fait cette formule ne figure pas littéralement dans la fable; nous utilisons peut-être un peu trop extensivement la notion d'îlot textuel ; mais on rattache bien au discours rapporté des citations non littérales!

${ }^{16}$ Nous incluons ici les titres à nom propre, qui évidemment n’impliquent pas un choix de déterminant.

17 «Un principe de base : la référence nécessite un calcul (Galmiche, 1989 : 22). 


\subsubsection{L'article défini singulier}

a 45 titres au singulier désignent un être animé qui est le personnage central de la fable.

Celui-ci est un animal le plus souvent, parfois un humain, une seule fois un objet - Le Cierge (IX, 12)-, une seule fois une allégorie - La Discorde (VI, 20). Nous pouvons mettre à part ce cas où l'article défini est à l'origine celui qui s'attache aux abstractions.

On sait que l'article défini est susceptible d'être employé pour un référent générique ou spécifique. Un SN seul ne peut-être qualifié de générique ou spécifique. L'interprétation de la plupart de nos titres ne peut donc se faire que rétroactivement. Par ailleurs le caractère narratif du texte soulève une contradiction, car les phrases génériques expriment normalement des vérités intemporelles. À l'inverse, une lecture spécifique est quelque peu décalée par rapport à l'orientation globale de la fable vers les généralités. (II, 17) :

Il faut bien cependant caractériser la différence entre Le Paon se plaignant à Junon

Le Paon se plaignait à Junon :

Déesse, disait-il, ce n'est pas sans raison

Que je me plains, que je murmure :

Le chant dont vous m'avez fait don

Déplait à toute la Nature... (vv. 1-5)

et Le Cerf se voyant dans l'eau (VI, 9) :

Dans le cristal d'une fontaine

Un Cerf se mirant autrefois ...

Nous pensons que la première option témoigne de l'ancrage de la fable dans le discours mythique et l'exemple le montre, qui pourrait être complété par un certain nombre d'autres dans lesquels les animaux sont les représentants de leur espèce toute entière. Nous aurons l'occasion plus loin de citer Les Grenouilles qui demandent un Roi (III, 4) et La Besace (I, 7). Nous parlerons ici de lecture archétypique.

La définitude est en cas ambivalente: unicité de l'être mythique, extension maximale dans la mesure où l'animal représente son espèce.

On peut noter aussi que le choix entre les deux options peut ne pas être homogène, mais varier selon les personnages. C'est le cas pour Le Lion (XI, 1), dont le héros éponyme est spécifique, mais les autres personnages archétypiques. La fable en effet met en scène Sultan Léopard qui « jadis » était tout puissant; or « Il naquit un lion dans la forêt voisine ». Le Renard, vizir du Léopard, incite celui-ci à s’en méfier : "J'ai fait son horoscope : il croîtra par la guerre ; /Ce sera le meilleur Lion/Pour ses amis qui soit sur terre (vv. 23-25). Le titre renverrait donc à cet individu. Cependant, l'animal étant la métaphore habituelle du roi, on peut penser à cette définitude 
particulière qui est celle des personnes assumant une fonction institutionnelle, comme un roi ou un pape ${ }^{18}$.

Quand la lecture est spécifique, par exemple dans Le Singe (XII, 19), l'article anticipe sur le récit, qui commence ainsi : «Il est un Singe dans Paris/A qui l'on avait donné femme. » De même dans Le Charlatan (VI, 19), dont les premiers vers sont :

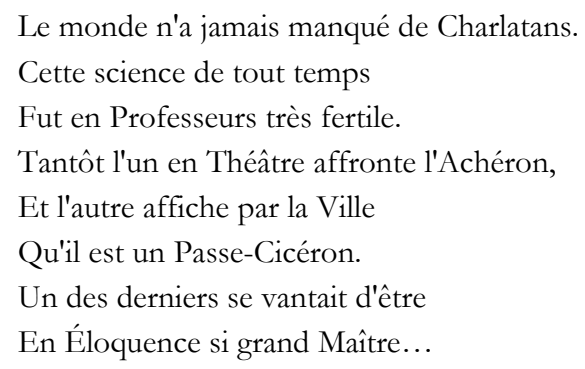

Nous nous sommes demandé si les éléments périphériques du titre pouvaient peser sur la lecture.

On a vu que ce n'était pas le cas du participe présent. C'est tout à fait normal, dans la mesure où celui-ci est un élément de type prédicatif. Il résume sans ancrage temporel tout ou partie du récit.

Le participe passé est susceptible de contribuer à la détermination et éventuellement à la définitude. Par ailleurs, il connote la narrativité parce qu'il comporte l'aspect accompli. Aussi la lecture des titres reste ambiguë : le titre peut être considéré comme thématique dans son ensemble et le participe serait définitoire, ou comme comportant un thème suivi d'un prédicat et le participe joue ce rôle. C'est plutôt la seconde configuration qui s'impose. On peut en conséquence poser en fin de compte que le participe passé est aussi neutre que le participe présent. De fait, par exemple, on a une lecture archétypique ${ }^{19}$ dans Le Lion devenu vieux (III, 14) :

Le Lion, terreur des forêts,

Chargé d'ans, et pleurant son antique prouesse,

Fut enfin attaqué par ses propres sujets,

Devenus forts par sa faiblesse. (vv. 1-4)

et une lecture spécifique dans Le Loup devenu Berger (III, 3) :

Un Loup qui commençait d'avoir petite part

Aux Brebis de son voisinage,

Crut qu'il fallait s'aider de la peau du Renard

Et faire un nouveau personnage.

Les syntagmes prépositionnels compléments de nom sont très actifs dans la détermination. Nous en avons deux de nature topographique : Le Paysan du Danube (XI, 7) et La Matrone d'Éphèse (XII, 26). Ce n'est cependant que par le récit que le

18 On est ici dans la zone des « désignateurs non-rigides » des logiciens (voir Galmiche, $1989: 8$ ).

${ }^{19}$ On peut toutefois s'interroger, dans la mesure où ce lion a une biographie... 
personnage s'érige en individu, car le Danube a plus d'un paysan et Ephèse plus d'une matrone! Cependant l'intertextualité pétronienne rappelée plus haut permet sans doute d'individualiser par avance cette matrone.

Les propositions relatives, dans le cadre de l'unité syntagmatique du titre, non séparées de l'antécédent par une virgule, sont nécessairement lues comme déterminatives ; on est donc bien dans le schéma canonique, le $N$ qui... toutefois cette structure ne prédispose pas nécessairement à la spécificité. Par ailleurs on pourrait aussi considérer que la relative fonctionne comme rhème.

Un exemple parmi les sept titres : L'Astrologue qui se laisse tomber dans un puits (II, 13) qui commence ainsi :

Un Astrologue un jour se laissa choir

Au fond d'un puits...

b Dans La Cour du Lion (VII, 6), nous avons un nom collectif qui désigne les personnages du récit, eux-mêmes définis par la relation avec le lion. L'article défini de cour s'impose donc, puisqu'un lion n'a qu'une cour ; quant à celui de lion, il nous semble suggérer pour le personnage le statut archétypique, peutêtre parce que la définitude est présupposée.

c Dans L'CEil du Maître (IV, 21), la définitude de xil vient du complément déterminatif et son singulier d'une sorte de synecdoque. Le maitre est spécifique dans le récit lui-même, mais dans la morale et ici, il est plutôt archétypique. Par ailleurs l'expression était proverbiale ${ }^{20}$ et on se rapproche peut-être ici d'une vraie généricité.

d Un titre - La Discorde (VI, 20) - désigne une abstraction, ce qui impose l'article défini, mais celle-ci, plutôt qu'un thème, est le personnage allégorique central de la fable.

e Un titre - L'Éducation (VIII, 24) - également abstrait, renvoie au sujet traité par le récit: deux chiens de même lignée, mais élevés dans des environnements différents deviennent l'un Laridon, l'autre César.

$f$ Deux titres renvoyant aussi à la thématique du récit ont leur article surdéterminé par la présence du complément de nom : Le pouvoir des fables (VIII, 4) , L'avantage de la Science (VIII, 19).

$g \quad$ L'article défini de La Besace (I, 12) est également surdéterminé, puisqu'il s'agit de la besace dont il va être question dans la morale mais aussi que chacun en porte une seule.

En effet, à la fin du récit où Jupiter demande à chacun des animaux s'il est satisfait de son physique, le fabuliste conclut :

\footnotetext{
${ }^{20}$ On trouve dans la première édition du Dictionnaire de l'Académie (1694) : «On dit prov. que L'œil du maistre engraisse le cheval, pour dire, que Quand un maistre a soin de prendre garde à ce qui se passe dans son domestique, tout en va mieux ». On peut être pratiquement sûr que le statut phraséologique de cette collocation préexistait à la fable, puisque P. Charron, en 1601, fait allusion à ce même proverbe (Source : Frantext, ATILF-CNRS).
} 
Jacques Anis

Le Fabricateur souverain

Nous créa Besaciers tous de même manière,

Tant ceux du temps passé que du temps d'aujourd'hui.

Il fit pour nos défauts la poche de derrière

Et celle de devant pour les défauts d'autrui. (vv. 31-35)

b Deux titres désignent un procès événementiel qui est au centre du récit, la définitude se fondant à la fois sur la singularité narrative et la présence d'un complément déterminatif : Le Songe d'un Habitant du Mogol (XI, 4) et Le combat des Rats et des Belettes (IV, 6).

\subsubsection{L'article défini pluriel}

a Les titres désignant les personnages principaux

Nous trouvons d'abord deux titres du type Les $N$ sec, Les Médecins $(\mathrm{V}, 12)$ et Les Devineresses (VII, 14). On découvre à la lecture que ces noms ont des référents spécifiques, la définitude venant donc de l'anticipation du texte. Dans les deux cas, deux personnages exercent la même profession. Citons le début de V, 12 :

Le Médecin Tant-pis allait voir un malade

Que visitait aussi son confrère Tant-mieux;

Ce dernier espérait, quoique son camarade

Soutînt que le gisant irait voir ses aïeux.

Cinq titres avec un numéral - par ex. Les deux Coqs (VII, 12) - relèvent de la même logique, mais le numéral associé au défini force au niveau du titre lui-même la lecture spécifique.

Nous avons deux titres mettant en scène des entités vraiment génériques: Les Animaux malades de la Peste (VII, 1) et Les Dieux voulant instruire un fils de Jupiter (XI, 2). On notera que l'adjectif comme le participe présent ont un statut purement prédicatif. Par ailleurs dans la première de ces fables, les animaux cités sont archétypiques.

Nous avons enfin deux titres composés de noms relationnels et collectifs déterminés par des N2, qui de surcroît sont des noms propres : Les filles de Minée (XII, 28) et Les Compagnons d'Ulysse (XII, 1). Il s'agit de véritables descriptions définies.

$b \quad$ Un titre désigne le procès qui est au centre du récit: Les obsèques de la Lionne (VIII, 14).

Le N1 appartient bien sûr à la catégorie des pluralia tantum. Le N2 est archétypique et nous trouvons à ce titre une certaine similitude avec La Cour du Lion (VII, 6). La fable met cependant en lumière des flottements entre l'archétypique et le spécifique, et il y a même une occurrence de lions qui pourrait être considérée comme véritablement générique :

La femme du Lion mourut :

Aussitôt chacun accourut

Pour s'acquitter envers le Prince

De certains compliments de consolation, 
Qui sont surcroît d'affliction. [...]

Le Prince aux cris s'abandonna,

Et tout son antre en résonna.

Les Lions n'ont point d'autre temple. (vv. 1-4 et 14-16)

\section{Conclusion}

En avançant dans le travail, nous avons mesuré la richesse du sujet de recherche et ressenti des frustrations. Ayant dû nous limiter aux titres uninucléaires, nous n'avons pas étudié le type le plus représentatif des titres, le binucléaire (La Cigale et la Fourmi, I, 1) et nous n'avons pas pu aborder, notamment, l'ordre des composants.

Cependant nous avons pu aboutir à quelques résultats intéressants :

- l'existence d'une syntaxe particulière des titres, dont nous menons l'analyse détaillée par ailleurs ;

- la complexité des interactions entre le titre et le texte dans la dynamique de la lecture et de la mémorisation;

- la multidimensionalité de la définitude ;

- l'absence de frontières tranchées entre le générique et le spécifique et l'existence d'un troisième terme que nous avons dénommé provisoirement archétypique ;

Nous pensons avoir entrevu et fait entrevoir au lecteur, à travers le premier et le dernier point, la primauté du texte sur les niveaux inférieurs ${ }^{21}$ et notamment la prégnance du genre.

Jacques ANIS

290, avenue d'Argenteuil

92600 Asnières

jacques.anis@u-paris10.fr

21 «Le palier du texte est primordial, puisque c'est la connaissance des caractéristiques du texte qui permet d'assigner du sens à la phrase et au mot. » (Rastier et al. 1994 : 36) 
Jacques Anis

\section{BIBLIOGRAPHIE}

ANIS J., 2002, «Le nom éponyme comme matrice textuelle dans le dossier des Cinq Sapates de Francis Ponge », Langages, 148, "Processus d'écriture et traces linguistiques", I. Fenoglio et S. Boucheron (éds), Larousse, Paris.

AUTHIER-REVUZ J., 1996, «Remarques sur la catégorie de l'îlot textuel », Cahiers du français contemporain 3, Credif, Didier, Paris : 91-115.

BOSREDON B., 1997, Les titres de tableau. Une problématique de l'identification, Paris, PUF.

GALMICHE M., 1979, «Quelques remarques sur l'exploitation linguistique de la notion de description définie », LINX, $1: 1-78$.

GALMICHE M., 1983, «L'utilisation des articles génériques comme mode de donation de la vérité », LINX, 9 : 29-87.

GALMICHE M., 1985, «Phrases, syntagmes et articles génériques », Langages, 79: 2-39, Larousse, Paris.

GALMICHE M., 1989, «A propos de la définitude », Langages, 94 : 7-37.

GALMICHE M. \& KLEIBER G., 1985, « Générique et généricité », Langages, 79.

GENETTE G, 1982, Palimpsestes. La Littérature au second degré, Seuil, Paris.

GUILLAUME G., 1919 (rééd. 1975), Le problème de l'article et sa solution dans la langue française, Paris et Québec, Nizet et PUQ.

RASTIER, F., CAVAZZA, M., ABEILLÉ, A., 1994, Sémantique pour l'analyse. De la linguistique à l'informatique, Masson, Paris.

RIEGEL M., PELLAT J.-C, RIOUL R., 1997, Grammaire méthodique du français, 2e édition, Paris, Presses Universitaires de France.

RIFFATERRE M., 1979, La production du texte, Paris, Seuil.

RIFFATERRE M., 1983, Sémiotique de la poésie (trad. fr. de Semiotics of poetry, 1978), Paris, Seuil.

SEARLE J., 1972, Les actes de langage. Essai de philosophie du langage, Paris, Hermann.

Éditions des Fables de La Fontaine

1) édition contemporaine servant de base à l'étude :

[1990] Fables choisies: mises en vers / La Fontaine ; [éd. par Georges Couton,...]. Numérisation BNF de l'éd. de Paris : Bibliopolis, 1998-1999. Reprod. de l'éd. de Paris : Bordas, 1990.

2) éditions consultées :

[1678-1694] Fables choisies : mises en vers par M. de La Fontaine et par lui-même revues, corrigées et augmentées... ; fig. gravées par François Chauveau Num. BNF de l'éd. de Paris : D. Thierry et $\mathrm{C}$. Barbin, 1678 pour les première, deuxième et troisième parties ; idem, 1679 pour la quatrième ; C. Barbin, 1694 pour la quatrième.

[1872] Fables / La Fontaine. II / travail de critique et d'érudition, aperçus d'histoire littéraire,... par Louis Moland Num. BNF de l'éd. de Liechtenstein : Kraus reprint, 1973. Reprod. de l'éd. de Paris : Garnier frères, 1872.

[1966] Fables, Garnier-Flammarion, Paris. 